\title{
Estudo físico-químico do fruto Garcinia mangostona L. e o desenvolvimento de uma farinha a partir do exocarpo
}

\section{Physico-chemical study of the fruit Garcinia mangostona L. and the development of a flour from the exocarp}

\author{
Danielle Castro da Silva ${ }^{1}$, Anne do Socorro Santos da Silva ${ }^{2}$, Antonio Carlos Souza da \\ Silva Júnior ${ }^{3}$
}

${ }^{1}$ Nutriocionista pelo Instituto Macapaense do Melhor Ensino Superior. Macapá-AP Brasil. E-mail: dhany29@hotmail.com *Autor para correspondência

${ }^{2}$ Pesquisadora do Instituto de Pesquisas Científicas e Tecnológicas do Estado do Amapá IEPA. Macapá-AP Brasil. E-mail: annedosocorro@hotmail.com

${ }^{3}$ Pesquisador do Instituto de Pesquisas Científicas e Tecnológicas do Estado do Amapá IEPA. Macapá AP Brasil. E mail: jr_bio2005@yahoo.com.br

\section{Palavras-chave}

Mangostão

Antioxidante

Fitoquímico

Flavonóide

\section{Keywords}

Mangosteen

Antioxidant

Phytochemical

Flavonoid
Originária da Ásia o mangostão, Garcinia mangostona L. é um fruto globoso, de exocarpo vermelho-púrpuro representando aproximadamente $70 \%$ de seu peso e mesocarpo branco-translúcido de sabor doce e agradável. O mangostão é conhecido no local de origem pelo potencial fitoterápico utilizado na medicina popular para tratar distúrbios gastrintestinais, infecções e ferimentos. Dentre os compostos bioativos presentes no fruto, principalmente no exocarpo, estão os flavonóides, antocianinas e xantonas. A comunidade científica tem relacionado estes fitoquímicos na terapêutica antioxidante, anti-inflamatória, antibacteriana, antiviral e antiproliferativa. O objetivo do trabalho foi caracterizar físico-quimicamente do fruto Garcinia mangostona e o desenvolvimento de uma farinha a partir do exocarpo. Para produção da farinha o exocarpo foi seco em estufa, triturado em moinho de rotor e armazenado; determinou-se proteínas pelo método Kjeldahl; cinzas, umidade e carboidratos segundo Instituto Adolfo Lutz; lipídeos pelo método de Bligh e Dyer; vitamina C pelo método de Tillmans; doseamento de antocianinas e flavonóides por Francis (1982); análises microbiológicas segundo Silva et al. (2010). A farinha revelou 5,11\%, 2,1\%, 22,04\% para proteínas, cinzas e umidade respectivamente, considerados bons resultados. Obteve-se $104,83 \mathrm{mg}$ de flavonóides, antocianinas $21,33 \mathrm{mg}$ e vitamina C $188 \mathrm{mg}$, sendo julgados teores excelentes. O produto apresentou alto valor calórico de 304,22kcal e seguro sanitariamente. A farinha exibiu resultados muito satisfatórios, podendo ser inserido na alimentação e auxiliar na manutenção da saúde.

Originally from Asia the mangosteen, Garcinia mangostona L. is a globose fruit, with red-purple exocarp representing approximately $70 \%$ of its weight and white-translucent mesocarp of sweet and pleasant flavor. Mangosteen is known at the site of origin for the herbal potential used in folk medicine to treat gastrointestinal disorders, infections and injuries. Among the bioactive compounds present in the fruit, mainly in the exocarp, are the flavonoids, anthocyanins and xanthones. The scientific community has linked these phytochemicals to antioxidant, anti-inflammatory, antibacterial, antiviral and antiproliferative therapy. The objective of this work was to characterize physically-chemically the fruit Garcinia mangostone and the development of a flour from exocarp. For the production of the flour the exocarp was dried in an oven, crushed in a rotor mill and stored; proteins were determined by the Kjeldahl method; ash, moisture and carbohydrates according to the Adolfo Lutz Institute; lipids by the method of Bligh and Dyer; vitamin C by the Tillmans method; assay of anthocyanins and flavonoids by Francis (1982); microbiological analyzes according to Silva et al. (2010). The flour showed 5.11\%, $2.1 \%, 22.04 \%$ for proteins, ashes and moisture respectively, considered good results. It was obtained $104.83 \mathrm{mg}$ of flavonoids, anthocyanins $21,33 \mathrm{mg}$ and vitamin C 188mg, being judged excellent contents. The product had a high caloric value of $304.22 \mathrm{kcal}$ and health insurance. The flour showed very satisfactory results, being able to be inserted in the food and to help in the maintenance of health.

\section{INTRODUÇÃO}

O mangostão possui origem asiática, pertence à família Clusiaceae e apresenta propagação bem limitada com afinidade às regiões tropicais, é cultivado especialmente na
Ásia, Índia e Austrália, tendo cultivo em poucos países da América (SACRAMENTO; COELHO JÚNIOR, 2005).

$\mathrm{Na}$ Bahia, pesquisadores da Embrapa Amazônia Oriental e da CEPLAC - Comissão Executiva do Plano da Lavoura Cacaueira - em 1976 despertaram interesse no mangostão, a 
partir de então o fruto foi ganhando espaço, sendo alvo de estudos e propagação no estado da Bahia, Amazônia e nos demais estados do Brasileiros (LEDERMAN et al., 2008).

O mangostão apresenta bom desenvolvimento em ares quentes e úmidos, não suportando à escassez de água e longos períodos de seca. O Brasil por exibir regiões em condições climáticas propícias para produção do mangostão, como no estado do Pará, acabou despertando interesse de descendentes de colonos japoneses no cultivo do fruto visando a sua exportação (MULLER et al., 1995).

Aproximadamente $70 \%$ do peso do mangostão é proveniente de sua casca, e a indústria alimentícia de segmento funcional tem atentado para alguns componentes presentes na casca desse fruto, que são os fitonutrientes denominados xantonas (LEDERMAN et al., 2008). Conforme Sacramento et al. (2007) o mangostão é consumido preferencialmente in natura, sua polpa representa uma média de $23,8 \%$ do seu peso, e que o fruto detém elevada ação antioxidante atribuído as xantonas, que atuam no organismo humano oferecendo vantagens à saúde.

A utilização de resíduos agroindustriais na criação de subprodutos direcionados ao consumo humano tem ganhado espaço no setor de tecnologia de alimentos, devido ao grande volume de resíduo gerado pelas indústrias, sendo que estes possuem significativos valores nutricionais, podendo ser reaproveitado (SILVA; ABE; SANTOS, 2013).

Em estudos realizados por Chisté et al. (2009) afirmaram que a casca do mangostão apresenta elevado teor de fibras totais, propondo a produção de farinha a partir da casca ou elaboração de outros produtos para indivíduos que demandam o consumo aumentado de fibras.

Experimentos realizados com extrato de Garcinia mangostona $\mathrm{L}$. constataram as características antioxidante, antitumoral, anti-inflamatória, antibacteriana e antiviral. Foi observado que a ingestão de frutas com expressiva quantidade de flavonoides também atuam no bom funcionamento do metabolismo energético através da formação de respostas inflamatórias, modificando fatores antropométricos e bioquímicos, ajudando na prevenção da obesidade (GOMES; SILVA; PINHEIRO-VOLP, 2016; CHAVERRI et al., 2008).

O estudo dos componentes físico-químicos presentes nos alimentos possui grande relevância na caracterização da sua funcionalidade e utilização, assim como para avaliar sua qualidade nutricional (CHISTÉ et al., 2009). Diante do exposto, este trabalho teve como objetivo desenvolver uma farinha a partir do exocarpo do fruto mangostão, Garcinia mangostona L., e realizar o estudo físico-químico tanto do fruto quanto da farinha produzido.

\section{METODOLOGIA}

Os frutos de mangostão foram obtidos diretamente do produtor com pomar na localidade de Campo Verde no município de Porto Grande no Estado do Amapá/AP, localizado na região Norte, latitude $00042^{\prime} 48^{\prime \prime} \mathrm{N}$, longitude 51 24' 48" W, altitude $60 \mathrm{~m}$ e área de $4421,6 \mathrm{Km}^{2}$. Para o desenvolvimento deste trabalho de pesquisa foram utilizados frutos da safra de maio de 2018. Onde foram transportados para o Instituto de Pesquisas Cientificas e Tecnológicas do Amapá (IEPA), Núcleo de ciência e Tecnologia de Alimentos, dispondo do Laboratório de Físico-Química de Alimentos, Laboratório de Alimentos Funcionais, Laboratório de Microbiologia, Usina de Óleo e Laboratório de Bromatologia do Instituto Macapaense do Melhor Ensino Superior (IMMES).

\section{Produção da farinha}

A obtenção da farinha do exocarpo de mangostão deu-se de acordo com o descrito abaixo em fluxograma (Figura 1):

Figura 1. Fluxograma de produção da farinha.

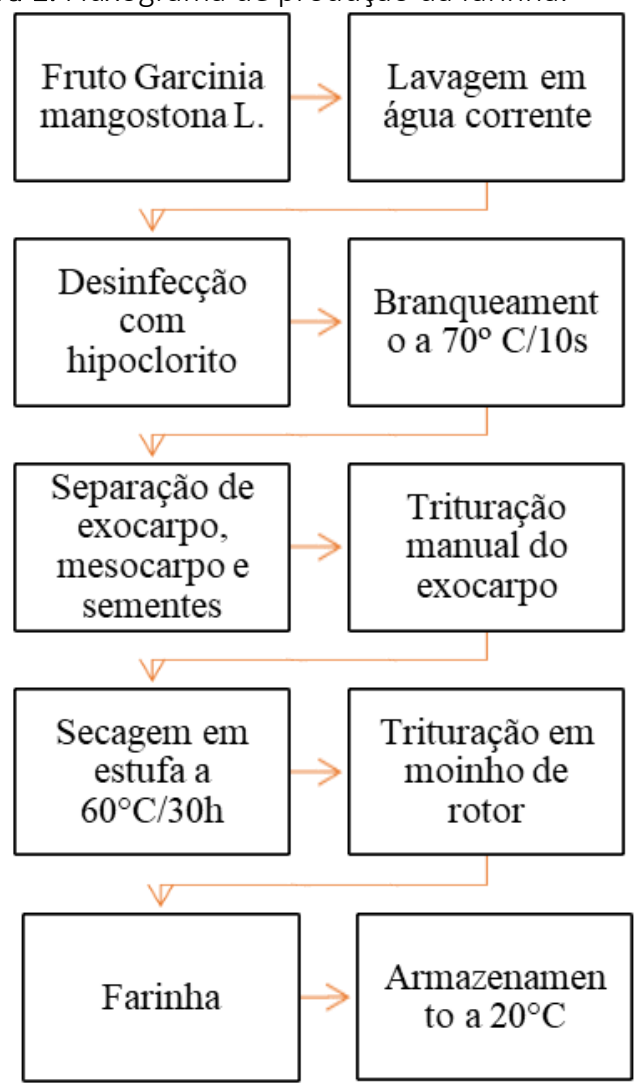

Foi realizada a lavagem e desinfecção do fruto (Figura 2) com $7,5 \mathrm{~mL}$ de hipoclorito à $200 \mathrm{ppm}$, utilizando uma colher de sopa para cada 1 litro de água deixando os frutos submersos durante 15 minutos, no branqueamento os frutos 
foram cobertos com água aquecida à 700 C por 10 segundos, retirados e transferidos para recipiente com água fria, em seguida deu-se a separação entre flor, exocarpo, mesocarpo e sementes sendo mantidos sob congelamento.

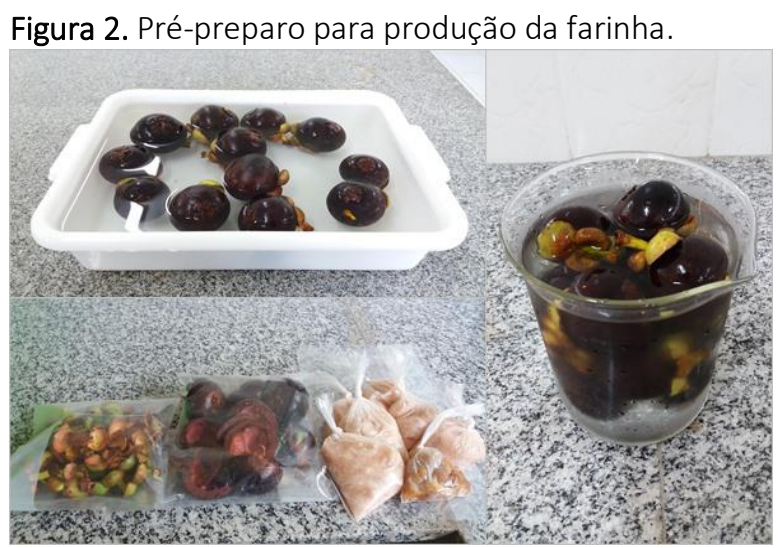

O exocarpo do mangostão foi cortado em pedaços menores, para facilitar a secagem e posteriormente sua trituração, após isso foi disperso em bandeja de aço inox e levado para estufa à 60 C por 30 horas consecutivas. Posteriormente a matéria seca foi moída em Moinho de rotor, marca Marconi, modelo MA 090/CFT (Figura 3). Sendo então obtido a farinha do exocarpo de Garcinia mangostona L., sem adição de qualquer classe de conservante. A farinha foi embalado em saco estéril, mantido à 20 $C$ ao abrigo de luz.

Figura 3. Obtenção da farinha.

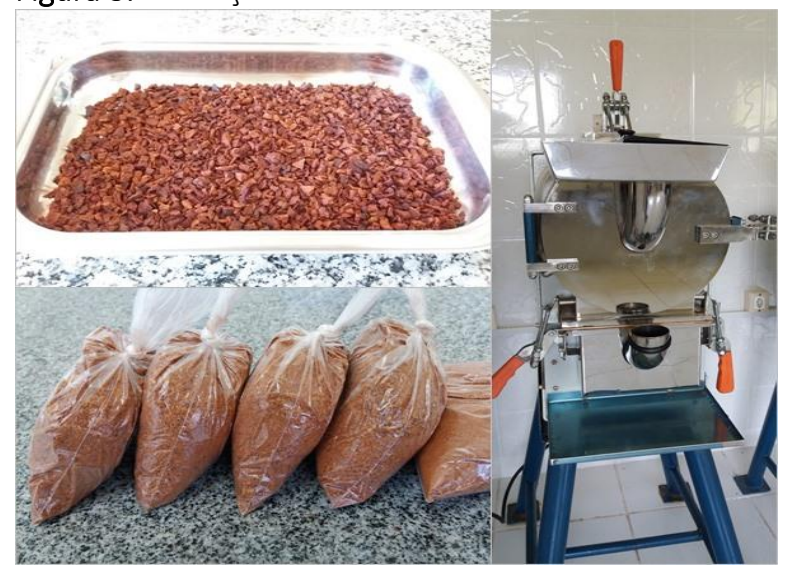

\section{Caracterização físico-química do fruto e farinha de Garcinia mangostona $L$.}

Todas as análises para determinação de proteínas, lipídeos, umidade, cinzas, vitamina C, flavonóides e antocianinas foram feitas em triplicata.

As proteínas foram determinadas pelo método de
Kjeldahl, através da digestão, destilação e titulação da amostra para determinação de nitrogênio total, utilizando aparelho da marca Tecnal, Modelo TE 036/1. Para determinação de resíduo mineral fixo (RMF) foi utilizado o método de calcinação em mufla da marca Quimis ${ }^{\circledR}$, modelo Q-318 D21, à temperatura de 550 C. Para determinação de umidade foi utilizada estufa da marca Quimis ${ }^{\circledR}$, modelo Q317 B222, utilizando o método de secagem em estufa com circulação de ar (INSTITUTO ADOLFO LUTZ, 2008).

Para determinação de lipídeos foi utilizado o método de Bligh e Dyer (1959) extração de lipídeos à frio. A determinação de carboidratos foi obtida pelo cálculo de diferenciação através da equação (1). Onde $E$ = carboidrato; $A=$ proteína total; $B=$ lipídeos; $C=$ umidade; $D=$ resíduo mineral fixo (INSTITUTO ADOLFO LUTZ, 2008). Para a quantificação do valor calórico total (VCT), prosseguiu-se utilizando a equação (8). Onde $E$ = carboidrato; $A$ = proteína total; $\mathrm{B}=$ lipídeos.

$$
\mathrm{VCT}=(\mathrm{Ex} 4)+(\mathrm{Ax} 4)+(\mathrm{Bx} 9)
$$

Para o doseamento de vitamina $C$, inicialmente foi realizado o preparo do extrato, onde foi pesada $5 \mathrm{~g}$ da amostra em Becker envolto em papel alumínio, acrescentouse $30 \mathrm{~mL}$ de ácido oxálico $0,5 \%$. Em seguida o extrato foi transferido para balão volumétrico de $100 \mathrm{~mL}$ envolvido em papel alumínio, sendo aferido o volume com solução de ácido oxálico e posteriormente o extrato foi levado à geladeira para conservação.

Para a determinação de vitamina C, tomando um Erlenmeyer de $125 \mathrm{~mL}$, foi adicionado $5 \mathrm{~mL}$ do extrato de vitamina C previamente preparado, mais $50 \mathrm{~mL}$ de água destilada. Em seguida foi titulado usando a solução refrigerada de Tillmans, titulando até que o extrato alcançasse coloração de tom rosado.

No doseamento de antocianinas e flavonóides, foi utilizado o método descrito por Francis (1982), pesado 1g de amostra em Becker envolvido em papel alumínio, e adicionado $\pm 30 \mathrm{~mL}$ da solução de etanol acidificado $(\mathrm{HCl} 1,5$ molar) previamente preparada. A mistura foi levada para agitador magnético marca Fisatom, modelo 761-5, por 5 minutos na velocidade " 5 ". Após isso, o conteúdo, não filtrado, foi transferido para balão volumétrico de $50 \mathrm{~mL}$, aferindo-se o volume com etanol acidificado.

$O$ extrato foi transferido para um frasco de vidro envolto em papel alumínio, e reservado em geladeira por uma noite. No dia seguinte prosseguiu-se a realização de leitura em espectrofotômetro UV-Vis, marca Kasuaki.

Na cubeta contendo o "branco" foi adicionado apenas a solução de etanol acidificado, nas demais cubetas o extrato anteriormente preparado. Para leitura de antocianinas 
utilizou-se comprimento de onda de $535 \mathrm{~nm}$ e para flavonóides $374 \mathrm{~nm}$. A quantificação de antocianinas deu-se através da equação (2). A quantificação de flavonóides foi obtido pela equação (3).

Absorbância x fator de diluição 98,2

Absorbância $x$ fator de diluiç̧̃̃o 76,6

\section{Análises microbiológicas}

Para análise de coliformes termotolerantes, pesou-se $10 \mathrm{~g}$ da amostra em Erlenmeyer adicionando $90 \mathrm{~mL}$ de água peptonada $\left(\mathrm{H}_{2} \mathrm{Op}\right)$, foi transferido $1 \mathrm{~mL}$ da solução para dois tubos contendo $9 \mathrm{~mL}$ de $\mathrm{H} 2 \mathrm{Op}$ e mais $1 \mathrm{~mL}$ para outros três tubos com Caldo Lauryl Sulfato Triptose (LST). Dos tubos contendo a amostra e H2Op também foram retirados $1 \mathrm{~mL}$ e transferindo-os para três tubos/cada com Caldo Lauryl Sulfato Triptose (LST). Todas as amostras foram incubadas a $35 \pm 0,2$ ㅇ $\mathrm{C}$ por $24-48 \mathrm{~h}$, sendo verificado se havia produção de gases ou turvação para dar prosseguimento as análises (SILVA et al., 2010).

Para a detecção da presença/ausência de Salmonella sp. foi pesado $10 \mathrm{~g}$ da amostra homogeneizada em $90 \mathrm{~mL}$ de Caldo Lactosado (STP) e levada para incubação a 35ㄷ durante $24 \mathrm{~h}$. Após isso foi transferido $100 \mu \mathrm{m}$ de STP para tubo contendo $10 \mathrm{~mL}$ de Caldo Rappaport-Vassilidis Soja (RVS) e incubados a $41,5 \pm 1$ 으 por $24 \pm 3 \mathrm{~h}$, sendo observado se as amostras apresentavam alguma alteração indicando crescimento de microrganismos, e assim prosseguir com as análises (SILVA et al., 2010).

\section{RESULTADOS E DISCUSSÃO}

Ao comparar os resultados obtidos da composição físicoquímica do mesocarpo de Garcinia mangostona L. (Tabela 1) observou-se umidade $84,49 \%$, semelhante ao descrito por
Braga; Assis Neto; Vilhena (2012), para proteínas obteve-se $1,97 \%$, sendo inferior a $3,12 \%$ dado pelos mesmos autores. Constatou-se que os valores para lipídeos e cinzas foram superiores aos achados por Braga; Assis Neto; Vilhena (2012) e Seixas; Virgolin; Janzantti (2017), acredita-se que as condições climáticas e tipos de solo em que os frutos foram adquiridos influenciaram diretamente na obtenção destes diferentes resultados. Quando quantificado o teor de vitamina C verificou-se que $93,33 \mathrm{mg}$ presente no mesocarpo do mangostão foi superior a 11,52 e $89,55 \mathrm{mg}$ dado por Seixas; Virgolin; Janzantti (2017) e Sousa et al. (2011) utilizando métodos e frutos diferentes, sendo considerado um excelente resultado tendo em vista que a Ingestão Diária Recomendada (DRI) para um adulto situa-se em até $90 \mathrm{mg} / \mathrm{dia}$.

Os resultados de proteínas e umidade 2,62\% e 64,02\% respectivamente, mostraram-se semelhantes aos de Chisté (2008), a média proteica também foi superior ao obtido por Xavier; Souza; Cândido (2015) devido a comparação entre frutos de gêneros diferentes. Quanto a determinação de cinzas $1,1 \%$ e lipídeos $2,22 \%$ foi muito superior ao encontrado por Chisté (2008) podendo ser atribuído a utilização de tratamentos diferenciados assim como fatores ambientais e climáticos. Com relação à antocianinas houve grande discrepância sendo 6,08mg bastante inferior a 25,94mg dado por Chisté (2008) supõe-se que o tempo de maturação do fruto assim como o armazenamento possam ter contribuído para a diferença entre os resultados. Freire et al. (2013) obteve $219 \mathrm{mg}$ de vitamina C no mesocarpo de caju, maior que 108mg no exocarpo de mangostão, esse resultado pode ser justificado pela dissemelhança de gêneros dos frutos assim como diferença na metodologia utilizada (Tabela 2).

As análises físico-químicas da farinha revelaram 5,11\%, 2,1\%, 22,04\% para proteínas, cinzas e umidade respectivamente, sendo maiores se comparados aos

Tabela 1. Caracterização físico-química de mesocarpos.

\begin{tabular}{lcccc}
\hline \multicolumn{1}{c}{ Análises } & $\begin{array}{c}\text { Autor } \\
\mathbf{( 2 0 1 8 ) *}\end{array}$ & $\begin{array}{c}\text { Braga; Assis Neto; } \\
\text { Vilhena (2012)* }\end{array}$ & $\begin{array}{c}\text { Seixas; Virgolin; } \\
\text { Janzantti (2017)** }\end{array}$ & Sousa et al. (2011)*** \\
\hline Proteínas (\%) & $1,97 \pm 0,28$ & $3,12 \pm 0,22$ & 2,82 & $1,65 \pm 0,26$ \\
\hline Lipídeos (\%) & $0,79 \pm 0,1$ & $0,17 \pm 0,18$ & 0,12 & $3,59 \pm 0,15$ \\
\hline Cinzas (\%) & $0,49 \pm 0,22$ & $0,10 \pm 0,04$ & 0,36 & $0,55 \pm 0,03$ \\
\hline Umidade (\%) & $84,49 \pm 0,46$ & $84,13 \pm 0,75$ & 89,67 & $83,45 \pm 0,06$ \\
\hline Vitamina C (mg) & 93,33 & - & 11,52 & 89,55 \\
\hline
\end{tabular}

* Garcinia mangostona L.; **Garcinia xanthochymus (mangostão amarelo); ***Resíduos de polpa de acerola (Malpighia glabra L.) 
descritos por Silva; Abe; Santos (2013) e Catarino (2016) acredita-se que tais diferenças entre estes resultados deramse pela utilização de metodologias distintas e adaptadas para cada produto elaborado. A farinha apresentou valores inferiores com relação a lipídeos $0,23 \%$ e antocianinas 21,33mg (Tabela 3), pressupõe-se que o estágio de maturação, período de armazenamento e temperatura a que matéria-prima foi submetida possa ter causando a diminuição desses nutrientes.

Verificou-se na farinha $188 \mathrm{mg}$ de vitamina C acima do quantificado por Clemente et al. (2012), importante resultado para o produto tendo em vista que o fruto da laranja é tido como referência em teores de AA. O produto exibiu 70,19 kcal de carboidratos maior que 32,1 kcal dado por Silva; Abe; Santos (2013), e menor que 88,36 kcal encontrado por Catarino (2016) podendo ser atribuído a diferença entre o gênero dos exocarpos. Quanto ao VET de $304,22 \mathrm{kcal}$ foi aumentado em relação aos demais autores que justifica-se pelo elevado aporte proteico e glicídico do fruto.

Na comparação de resultados entre o exocarpo in natura e a farinha foi alcançado boa redução referente a umidade passando de $64,02 \%$ para $22,04 \%$ garantindo maior conservação e durabilidade do produto. A farinha apresentou $5,11 \%$ referente a proteínas, sendo superior a $2,62 \%$ no exocarpo in natura, acredita-se que a perda de umidade tenha favorecido a concentração de aminoácidos. Em relação a lipídeos houve perda considerável de 2,22\% para $0,23 \%$ contido na farinha, supõe-se que devido ao período de secagem em estufa. Na Figura 4 estão expressos os valores médios referentes ao fruto e produto final.

Comparando os resultados para antioxidantes tanto do fruto in natura quanto do produto final (Tabela 4), constatouse que não ocorreram perdas concernente a flavonóides, antocianinas e vitamina $C$, pelo contrário a farinha apresentou um aumento em relação a estes componentes, acredita-se ter havido concentração destes nutrientes após a secagem assim como maior precaução no armazenamento

Tabela 2. Caracterização físico-química de exocarpos in natura.

\begin{tabular}{|c|c|c|c|c|}
\hline Análises & Autor (2018)* & Chisté (2008)* & $\begin{array}{c}\text { Xavier; Souza; } \\
\text { Cândido (2015)** }\end{array}$ & $\begin{array}{l}\text { Freire et al. } \\
(2013)^{* * *}\end{array}$ \\
\hline Proteínas (\%) & $2,62 \% \pm 0,54$ & $2,55 \% \pm 0,06$ & 1,65 & - \\
\hline Lipídeos (\%) & $2,22 \% \pm 0,05$ & $0,72 \% \pm 0,07$ & - & - \\
\hline Cinzas (\%) & $1,1 \% \pm 0,38$ & $0,47 \% \pm 0,05$ & 1,18 & - \\
\hline Umidade (\%) & $64,02 \% \pm 0,62$ & $64,51 \% \pm 0,16$ & 87,75 & - \\
\hline Antocianinas (mg) & $6,08 \mathrm{mg}$ & $25,94 \mathrm{mg}$ & - & - \\
\hline Vitamina C (mg) & $108 \pm 14,42$ & - & - & $219 \pm 29,48$ \\
\hline \multicolumn{5}{|c|}{$\begin{array}{l}\text { *Exocarpo de mangostão; } * * \text { Exocarpo maracujá amarelo; } * * * \text { Polpa de caju in natura } \\
\text { Tabela 3. Caracterização físico-química da farinha de mangostão e outros produtos a }\end{array}$} \\
\hline Análises & Autor (2018)* & $\begin{array}{c}\text { Silva; Abe; Santos } \\
(2013)^{* *}\end{array}$ & Catarino $(2016)^{* * *}$ & $\begin{array}{l}\text { Clemente et al. } \\
\qquad(2012)^{* * * *}\end{array}$ \\
\hline Proteínas (\%) & $5,11 \pm 0,49$ & $3,0 \pm 0,04$ & $4,44 \pm 0,27$ & $11,08 \pm 0,4$ \\
\hline Lipídeos (\%) & $0,23 \pm 0,01$ & $1,3 \pm 0,14$ & $1,35 \pm 0,01$ & - \\
\hline Cinzas (\%) & $2,1 \pm 0,17$ & $1,5 \pm 0,33$ & $5,85 \pm 0,19$ & - \\
\hline Umidade (\%) & $22,04 \pm 1,83$ & $10,03 \pm 0,48$ & $9,20 \pm 0,18$ & $0,96 \pm 0,06$ \\
\hline Antocianinas (mg) & 21,33 & 41,9 & - & - \\
\hline Vitamina C (mg) & $188 \pm 28,84$ & - & - & $145,83 \pm 2,2$ \\
\hline Carboidratos (kcal) & $70,19 \pm 2$ & $32,1 \pm 0,15$ & 88,36 & - \\
\hline VET (kcal) & 304,22 & 146,2 & - & - \\
\hline
\end{tabular}

\footnotetext{
* Farinha; ** farinha da exocarpo de mangostão; *** farinha da exocarpo do maracujá; ****farinha de resíduos de laranja
} 
dos resíduos destinados a farinha.

O fitoquímico flavonóide mostrou-se elevado no fruto Garcinia mangostona L. desde resultados de seu mesocarpo de com 9,06mg. Quando verificado o teor de flavonóides na farinha obteve-se $104,83 \mathrm{mg}$ sendo superior a 52,69mg encontrado por Arcanjo (2015) em vinhos tintos. A farinha também apresentou $21,33 \mathrm{mg}$ de antocianinas, maior que 14,23 e 15,35mg referidos por Valente et al. (2017) e Arcanjo (2015) respectivamente (Tabela 5). Dessa forma os resultados foram muito satisfatórios visto que o fruto do açaí e da uva com o qual a farinha foi comparado são conhecidamente detentores de altos teores de flavonóide e antocianinas.

Quando realizada as análises microbiológicas da farinha os resultados revelaram ausência de crescimento de microrganismos, podendo ser explicado pelo método de prétratamento assim como pelo tempo de secagem a que a matéria foi submetida, certificando que os procedimentos de manipulação foram eficazes (Tabela 6).

\section{CONCLUSÕES}

O estudo constatou que a farinha produzido a partir do exocarpo de Garcinia mangostona L. destacou-se no quesito antioxidantes exibindo teores excelentes para flavonóides, antocianinas e vitamina C. Sabe-se que os compostos antioxidantes são muito importantes pela sua funcionalidade no organismo protegendo-o principalmente no combate à radicais livres, a qual um indivíduo está exposto constantemente. Cientistas vem relacionando o consumo de alimentos ricos em antioxidantes à manutenção da saúde, prevenção de doenças e qualidade de vida. Assim essas substancias vem sendo estudadas e empregadas em
Tabela 4. Médias dos resultados para antioxidantes do exocarpo in natura e farinha do exocarpo do mangostão.

\section{Análises Exocarpo Farinha}

\begin{tabular}{lcc}
\hline Flavonóides (mg) & $101,64 \pm 0,07$ & $104,83 \pm 0,42$ \\
\hline Antocianinas (mg) & $6,08 \pm 0,19$ & $21,33 \pm 2,24$ \\
\hline Vitamina C (mg) & $108 \pm 14,42$ & $188 \pm 28,84$
\end{tabular}

Tabela 5. Comparação de resultados para antocianinas e flavonóides da farinha do exocarpo do mangostão

\begin{tabular}{llcc}
\hline \multicolumn{1}{c}{ Análises } & $\begin{array}{c}\text { Autor } \\
\mathbf{( 2 0 1 8 )}\end{array}$ & $\begin{array}{c}\text { Valente et } \\
\text { al. (2017)** }\end{array}$ & $\begin{array}{c}\text { Arcanjo } \\
\mathbf{( 2 0 1 5 )} * * *\end{array}$ \\
\hline Flavonóides & 104,83 & - & 52,69 \\
\hline Antocianinas & 21,33 & 14,23 & 15,35 \\
\hline $\begin{array}{l}* \text { farinha exocarpo } \\
* * * \text { mangostão; }\end{array}$ & **corante de açaí em pó; \\
\end{tabular}

Tabela 6. Resultados de análises microbiológicas do Farinha

Análises $\begin{aligned} & \text { Farinha do Legislação* } \\ & \text { exocarpo de } \\ & \text { mangostão }\end{aligned}$

Coliformes $\quad<3 \mathrm{NMP} / \mathrm{g} \quad<10^{2} \mathrm{NMP} / \mathrm{g}$

termotolerantes

$35^{\circ} \mathrm{C}$

\section{Salmonella sp. Ausente em $10 \mathrm{~g} \quad$ Ausente}

Número mais provável para farinhas; * BRASIL (2001)

Figura 4. Comparação de resultados de médias do exocarpo in natura e da sua farinha.

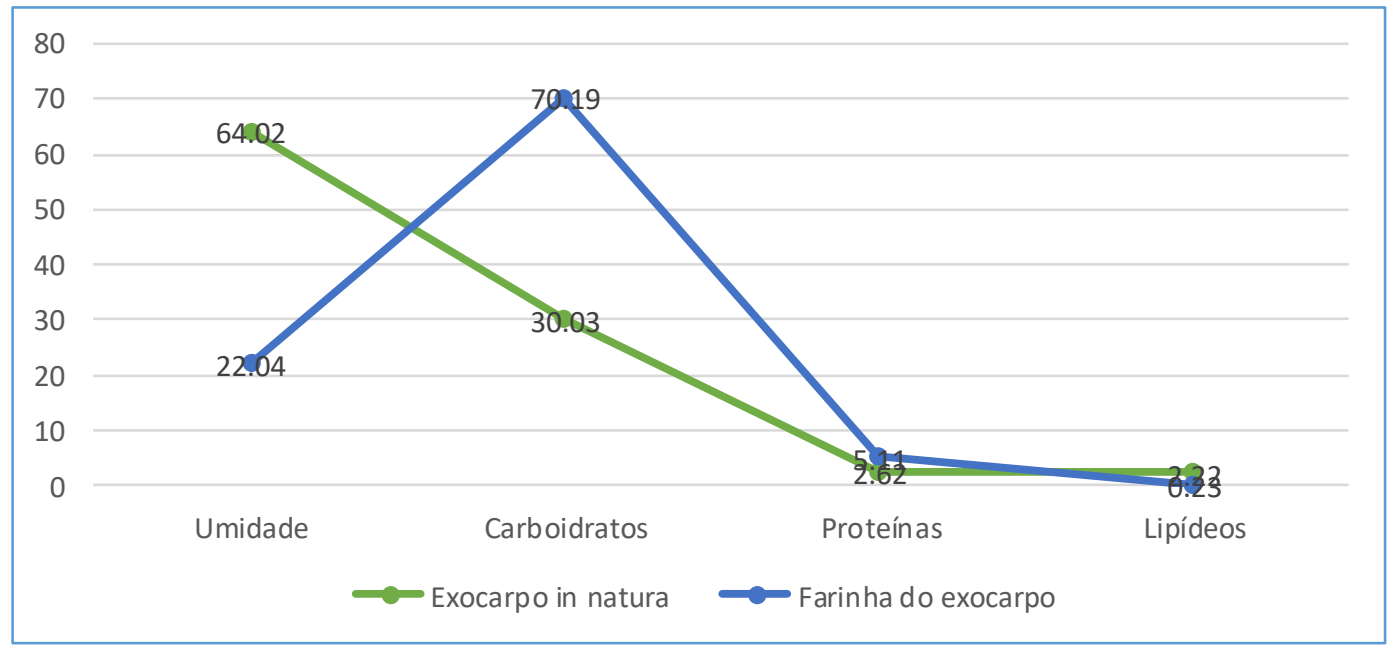


tratamentos contra doenças crônicas como diabetes e cânceres.

O produto final apresentou considerável porte proteico e alto valor calórico podendo ser utilizado como complemento e incorporado à refeições de indivíduos que demandem um maior gasto energético. Com relação a umidade da farinha o teor obtido foi muito satisfatório permitindo assim um produto sanitariamente seguro.

A farinha produzida expressa possibilidade de introdução na dieta alimentar humana sendo utilizado como colorífico e complemento em preparações diárias de alimentos contribuindo para o bom funcionamento do organismo. Dessa forma o reaproveitamento dos resíduos do exocarpo de mangostão, que comumente são descartados, receberiam um novo destino podendo alcançar lugar no mercado consumidor auxiliando na qualidade de vida.

\section{REFERÊNCIAS}

ARCANJO, N. M. O. Qualidade de vinho tinto produzido com uvas da cultivar isabel (Vitis labrusca) proveniente de duas regiões do Brasil (Noedeste e Sul). 2015. 135 f. Dissertação (Mestrado em Ciências e Tecnologia de Alimentos) - Universidade Federal da Paraíba, João Pessoa, 2015.

BRAGA, A. C. C.; ASSIS NETO, E. F.; VILHENA, M. J. V. Elaboração e caracterização de iogurtes adicionados de polpa e de xarope de mangostão (Garcinia mangostana L.). Revista Brasileira de Produtos Agroindustriais. v.14, n.1, p. 77-84, 2012.

CATARINO, R. P. F. Elaboração e caracterização de farinha de casca de maracujá para aplicação em biscoitos. 2016. 39 f. Trabalho de Conclusão de Curso (Graduação em Tecnólogo em Alimentos) - Universidade Tecnológica Federal do Paraná, Londrina, 2016.

CHAVERRI, J. P.; RODRÍGUEZ, N. C.; IBARRA, M. O.; ROJAS, J. M. P. Medicinal properties of mangosteen (Garcinia mangostana). Food and Chemical Toxicology. v.46, n.10, p.3227-3238, 2008.

CHISTÉ, R. C. Estabilidade do extrato antociânico obtido da casca de mangostão (Garcinia mangostana I.). 2008. 106 f. Dissertação (Mestrado em Ciência e Tecnologia de Alimentos) - Instituto de Tecnologia, Universidade Federal do Pará, Belém, 2008.

CHISTÉ, R. C.; FARIA, L. J. G.; LOPES, A. S.; MATTIETTO, R. A. Características físicas e físico-química da casca de mangostão em três períodos da safra. Revista Brasileira de Fruticultura. v. 31, n. 2, p. 416-422, 2009.

CLEMENTE, E.; FLORES, A. C.; ROSA, C. I. L. F.; OLIVEIRA, D. M. Características da farinha de resíduos do processamento de laranja. Revista Ciências Exatas e Naturais. v. 14, n. 2, p. 257-269, 2012.

FRANCIS, F.J. Analysis of anthocyanins. In: MARKAKIS, P. Anthocyanins as food colors. London: Academic Press, 1982. p.181-206.

FREIRE, J. M.; ABREU, C. M. P.; ROCHA, D. A.; CORRÊA, A. D.; MARQUES, N. R. Quantificação de compostos fenólicos e ácido ascórbico em frutos e polpas congeladas de acerola, caju, goiaba e morango. Ciência Rural. v. 43 n. 12., p. 2291-2296, 2013.

GOMES, F. S.; SILVA, F. C.; PINHEIRO-VOLP, A. C. Effect of consumption of fruits rich in flavonoids on inflammatory, biochemical and anthropometric mediators related to energy metabolism. Nutrición clínica y dietética hospitalaria. v. 36, n. 3, p. 170-180, 2016.

INSTITUTO ADOLFO LUTZ. Procedimentos e Determinações Gerais. IN: ZENEBON, O.; PASCUET, N. S.; TIGLEA, P. (Org.). Métodos físico-químicos para análise de alimentos. 4. ed. São Paulo: Instituto Adolfo Lutz, 2008, cap. 4, p. 83-158.

LEDERMAN, I. E.; SILVA JUNIOR, J. F.; SACRAMENTO, C. K.; CARVALHO, J. E. U.; YAMANISHI, O. K. Frutas exóticas potenciais. IN: ALBUQUERQUE, A. C.; SILVA, E. (Org.). Agricultura Tropical: quatro décadas de inovações tecnológicas, institucionais e políticas. ed. Brasília: Embrapa Informação Tecnológica. v. 1, cap. 2, p. 437-460. 2008.

MULLER, C. H.; FIGUEIRÊDO, F. J. C.; NASCIMENTO, W. M. O.; CARVALHO, J. E. U.; STEIN, R. L. B.; SILVA, A. B.; RODRIGUES, J. E. L. F. A cultura do Mangostão. IN: ANDREOTTI, C. (Org.). Coleção Plantar. 28. ed. Brasília: EMBRAPA - SPI, 1995, 56 p.

SACRAMENTO, C. K.; COELHO JÚNIOR, E.; CARVALHO, J. E. U.; MÜLLER, C. H.; NASCIMENTO, W. M. O. Cultivo do mangostão no Brasil. Revista Brasileira de Fruticultura. v.29, n.1, p.195-203. 2007.

SACRAMENTO, C.K.; COELHO JÚNIOR, E. Cultivo do mangostão na Bahia. Bahia Agrícola. v. 7, n.1, p. 15-18. 2005.

SILVA, A. K. N.; ABE, S. T. H.; SANTOS, O. V. Processamento da farinha da casca do mangostão (Garcinia mangostana L.) com vistas aos aspectos nutricionais e de antocianina. Revista Brasileira de Tecnologia Agroindustrial. v. 07, n. 02, p. 1074- 1087. 2013.

SILVA, N.; JUNQUEIRA, V.C.A.; SILVEIRA, N.F.A.; TANIWAKI, M.H.; SANTOS, R.F.S.; GOMES, R.A.R. Manual de Métodos de Análise Microbiológica de Alimentos e Água. 4. Ed. São Paulo: Livraria Varela, 2010.

SOUSA, M. S. B.; VIEIRA, L. M.; SILVA, M. J. M.; LIMA, A. Caracterização nutricional e compostos antioxidantes em resíduos de polpas de frutas tropicais. Ciência e Agrotecnologia. v. 35, n. 3, p. 554-559. 2011. 
VALENTE, M.C.C.; SANTANA, D.; SANTANA, E.; COSTA, C.M.L. Estabilidade do corante de antocianinas extraídas do fruto de açaí (Euterpe oleracea Mart.). Anais.. IN: XXXVIII Congresso Brasileiro de Sistemas Particulados. 22 a 25 de outubro de 2017. Maringá - Paraná.

VIRGOLIN, L.B.; SEIXAS, F.R.F.; JANZANTTI, N.S. Composition, content of bioactive compounds, and antioxidant activity of fruit pulps from the Brazilian Amazon biome. Pesquisa Agropecuária Brasileira. v.52, n.10, p.933-941, 2017.

XAVIER, G.F.; SOUZA, B.S.; CÂNDIDO, T.A.T. Determinação da composição centesimal da casca e da farinha da casca de maracujá. Anais.. IN: 7ạ Jornada Científica e Tecnológica do IF Sul de Minas. 12 de Novembro de 2015. Poços de Caldas, Minas Gerais.

Submissão: 06/03/2019

Aprovado para publicação: 21/03/2019 